\title{
Botnet Detection using Social Graph Analysis*
}

\author{
Jing Wang ${ }^{\dagger}$ and Ioannis Ch. Paschalidis ${ }^{\ddagger}$
}

\begin{abstract}
Signature-based botnet detection methods identify botnets by recognizing Command and Control $(\mathrm{C} \& \mathrm{C})$ traffic and can be ineffective for botnets that use new and sophisticate mechanisms for such communications. To address these limitations, we propose a novel botnet detection method that analyzes the social relationships among nodes. The method consists of two stages: (i) anomaly detection in an "interaction" graph among nodes using large deviations results on the degree distribution, and (ii) community detection in a social "correlation" graph whose edges connect nodes with highly correlated communications. The latter stage uses a refined modularity measure and formulates the problem as a nonconvex optimization problem for which appropriate relaxation strategies are developed. We apply our method to real-world botnet traffic and compare its performance with other community detection methods. The results show that our approach works effectively and the refined modularity measure improves the detection accuracy.
\end{abstract}

Index Terms-Network anomaly detection, cyber-security, social networks, random graphs, optimization.

\section{INTRODUCTION}

A botnet is a network of compromised nodes (bots) controlled by a "botmaster." The most common type is a botnet of network computers, which is usually used for Distributed Denial-of-Service (DDoS) attacks, click fraud and spamming, etc. DDoS attacks comprise packet streams from disparate bots, aiming to consume some critical resource at the target and to deny the service of the target to legitimate clients. In a recent survey, 300 out of 1000 surveyed businesses have suffered from DDoS attacks and $65 \%$ of the attacks cause up to $\$ 10,000$ loss per hour [1]. Both click fraud and spamming are harmful to web economy. Click fraud exhausts the advertisement budgets of businesses in pay-per-click services [2], and spamming is popular for malicious advertisements as well as manipulation of search results [3].

Because of the huge loss caused by botnets, detecting them in time is very important. Most of the existing botnet detection approaches focus on Command and Control (C\&C) channels required by botmasters to command their bots [4], [5]. One mechanism is to filter specific types of C\&C traffic (e.g., IRC traffic) [6], [7], [8]. Recently, botnets have evolved to bypass these detection methods by using more sophisticated C\&C channels, such as HTTP and P2P protocols [9], [2]. P2P botnets like Nugache [10] and Storm

Research partially supported by the NSF under grants CNS-1239021 and IIS-1237022, by the ARO under grants W911NF-11-1-0227 and W911NF12-1-0390, and by the ONR under grant N00014-10-1-0952.

$\dagger$ Division of Systems Engineering, Boston University, 8 St. Mary's St., Boston, MA 02215, wangjing@bu.edu.

$\ddagger$ Dept. of Electrical \& Computer Eng., Boston University, 8 Mary’s St., Boston, MA 02215, yannispabu.edu. worm [9] are much harder to detect and mitigate because they are decentralized. In addition, more types of $\mathrm{C} \& \mathrm{C}$ channels are emerging; recent research shows that botnets start to use Twitter as the $\mathrm{C} \& \mathrm{C}$ channel [11]. It is very challenging to identify and monitor these sophisticated $\mathrm{C} \& \mathrm{C}$ channels. Furthermore, the switching cost of $\mathrm{C} \& \mathrm{C}$ channels is much lower than the monitoring cost, thus botnet can bypass detection by changing $\mathrm{C} \& \mathrm{C}$ channels frequently.

In addition to $\mathrm{C} \& \mathrm{C}$ channels, botnets have some behavioral characteristics. First, bots activities are more correlated with each other than normal nodes [12], [8]. Second, bots have more interactions with a set of pivotal nodes, including targets and botmasters. Compared with $\mathrm{C} \& \mathrm{C}$ traffic, these behavioral characteristics are harder to hide.

In this paper, we propose a novel botnet detection framework based on these behavioral characteristics. Instead of focusing on $\mathrm{C} \& \mathrm{C}$ channels, we detect botnets by analyzing the social relationships, modeled as graphs of nodes. Two types of social graphs are considered: (i) Social Interaction Graphs (SIGs) in which two nodes are connected if there is interaction between them, and (ii) Social Correlation Graphs (SCGs) in which two nodes are connected if their behaviors are correlated. We apply our method to real-world botnet traffic, and the results show that it has high detection accuracy.

\section{Method Overview}

We assume the data to be a sequence of interaction records; each record $\mathrm{r}=$ (timestamp, id1, id2) contains a timestamp and the IDs of the two participants. For botnets of network computers, a interaction record corresponds to a network packet.

We group interaction records into windows based on their timestamps. For all $k$ 's, we denote by $\mathcal{W}_{k}$ the collection of interaction records in window $k$ and present the definition of the Social Interaction Graph (SIG) for window $k$ as follows.

\section{Definition 1}

(Social Interaction Graph). Let $\mathcal{E}_{k}$ be an edge set such that $(i, j) \in \mathcal{E}_{k}$ if there exists at least one interaction record $\mathrm{r} \in \mathcal{W}_{k}$ whose participant IDs are $i$ and $j$. Then, the SIG $\mathcal{G}_{k}=\left(\mathcal{V}, \mathcal{E}_{k}\right)$ corresponding to $\mathcal{W}_{k}$ is an undirected graph whose vertex set $\mathcal{V}$ is the set of all nodes in the network and whose edge set is $\mathcal{E}_{k}$.

On a notational remark, throughout the paper we will use $n$ to denote the number of nodes in the network (cardinality of $\mathcal{V}$ ).

Our method consists of a network anomaly detection stage and a botnet discovery stage (see Fig. 11). In the 
network anomaly detection stage, each SIG is evaluated with a reference model and abnormal SIGs are stored into a pool $\mathcal{A}$. The botnet discovery stage is triggered whenever the size of the pool $\mathcal{A}$ is greater than a threshold $p$. A set of highly interactive nodes, referred to as pivotal nodes, are identified. Both botmaster and targets are very likely to be pivotal nodes because they need to interact with bots frequently. These interactions correspond to $\mathrm{C} \& \mathrm{C}$ traffic for botmasters and to attacking traffic for targets. In either case, the interactions between each bot and pivotal nodes should be correlated. To characterize this correlation, we construct a Social Correlation Graph (SCG), whose formal definition is in Section IV-B.1. We can detect bots by detecting the community that has high interaction with pivotal nodes in the SCG. We propose a novel community detection method based on a refined modularity measure. This modularity measure uses information in SIGs, i.e., pivotal interaction measure (see Section IV-B.3, to improve detection accuracy.

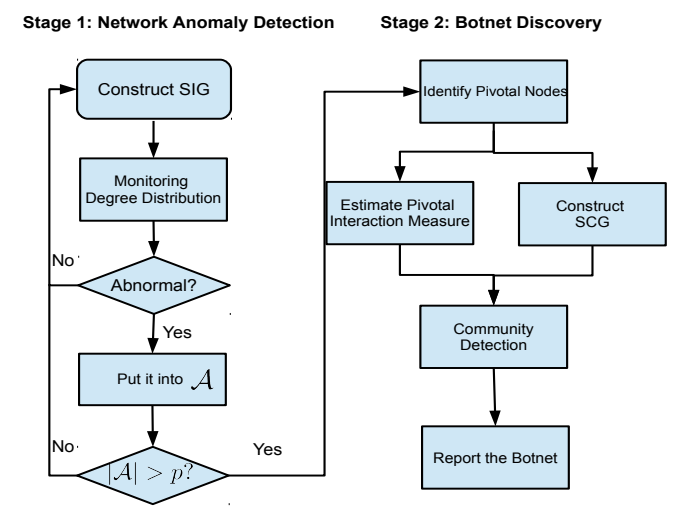

Fig. 1. Overview of Our Method.

\section{Network AnOmaly Detection}

As noted above, the goal of the network anomaly detection stage is to identify abnormal SIGs given some knowledge of what constitutes "normal" interactions between nodes. A natural way is to monitor the degree distributions of graphs and to compare them with appropriate reference graph models. This paper focuses on the Erdös-Rényi (ER) model, the most common type of random graph models. Our approach, however, can be generalized to more types of models. We apply composite hypothesis testing to detect abnormal graphs.

\section{A. Large Deviation Principle for ER Random Graphs}

First, we present a Large Deviation Principle (LDP) for undirected random graphs. Let $\mathcal{G}_{n}$ denote the space of all simple labeled undirected graphs of $n$ vertices. For any graph $\mathcal{G} \in \mathcal{G}_{n}$, let $\mathbf{d}=\left(d_{1}, \ldots, d_{n}\right)$ denote the labeled degree sequence of $\mathcal{G}$. Also let $m=\frac{1}{2} \sum_{j=1}^{n} d_{j}$ denote the number of edges in $\mathcal{G}$. We assume that any two nodes are connected by at most one edge, which means that the node degree in $\mathcal{G}$ is less than $n$. For $0 \leq i \leq n-1$, let $h_{i}=\sum_{j=1}^{n} 1\left(d_{j}=i\right)$ be the number of vertices in $\mathcal{G}$ of degree $i$, where $1(\cdot)$ is the indicator function. Henceforth, $\mathbf{h}=\left(h_{0}, \ldots, h_{n-1}\right)$, a quantify irrelevant to the ordering of vertices, will be referred to as the degree frequency vector of a graph $\mathcal{G}$. The empirical distribution of the degree sequence $\mathbf{d}$, defined by $\boldsymbol{\mu}^{(n)}$, is a probability measure on $\mathbb{N}_{0}=\mathbb{N} \cup\{0\}$ that puts mass $h_{i} / n$ at $i$, for $0 \leq i \leq n-1$.

In the Erdös-Rényi model, $\mathcal{G}(n, p)$, the distribution of the degree of any particular vertex $v$ is binomial. Namely, $P\left(d_{v}=k\right)=\left(\begin{array}{c}n-1 \\ k\end{array}\right) p^{k}(1-p)^{n-1-k}$, where $n$ is the total number of vertices in the graph. It it well known that when $n \rightarrow \infty$ and $n p$ is constant, the binomial distribution converges to a Poisson distribution. Let $\beta=n p$ denote the constant. Then in the limiting case, the probability that the degree of a node equals $k$ is $p_{\beta, k}=\frac{\beta^{k} e^{-\beta}}{k !}$, which is independent of the node label. Let $\mathbf{p}_{\beta}=\left(p_{\beta, 0}, \ldots, p_{\beta, \infty}\right)$ be the Poisson distribution viewed as a vector whose parameter is $\beta$.

Let $\mathbb{P}\left(\mathbb{N}_{0}\right)$ be the space of all probability measures defined on $\mathbb{N}_{0}$. We view any probability measure $\boldsymbol{\mu} \in$ $\mathbb{P}\left(\mathbb{N}_{0}\right)$ as an infinite vector $\boldsymbol{\mu}=\left(\mu_{0}, \ldots, \mu_{\infty}\right)$. Let $\mathcal{S}=$ $\left\{\boldsymbol{\mu} \in \mathbb{P}\left(\mathbb{N}_{0}\right): \bar{\mu}:=\sum_{i=0}^{\infty} i \mu_{i}<\infty\right\}$ be the set of all probability measures on $\mathbb{N}_{0}$ with finite mean. It is easy to verify that $\mathbf{p}_{\beta} \in \mathcal{S}$. Let $\mathbf{P}_{n}$ denote the Erdös-Rényi distribution on the space $\mathcal{G}_{n}$ with parameter $\beta / n$.

The so-called rate function $I: \mathcal{S} \rightarrow[-\infty, \infty]$ can be used to quantify the deviations of $\boldsymbol{\mu}^{(n)}$ with respect to a random graph model ([13], [14]). For the ER model, [13] proposes the following rate function.

\section{Definition 2}

For the ER model with parameter $\beta$ for its degree distribution, we could define the rate function $I_{E R}: \mathcal{S} \rightarrow[-\infty, \infty]$ as

$I_{E R}(\boldsymbol{\mu} ; \beta)=D\left(\boldsymbol{\mu} \| \mathbf{p}_{\beta}\right)+\frac{1}{2}(\bar{\mu}-\beta)+\frac{\bar{\mu}}{2} \log \beta-\frac{\bar{\mu}}{2} \log \bar{\mu}$, where $D\left(\boldsymbol{\mu} \| \mathbf{p}_{\beta}\right)=\sum_{i} \mu_{i} \log \left(\frac{\mu_{i}}{p_{\beta, i}}\right)$ is the Kullback-Leibler (KL) divergence of $\boldsymbol{\mu}$ with respect to $\mathbf{p}_{\beta}$.

[13] further establishes an LDP for $\boldsymbol{\mu}^{(n)}$ with this rate function. In the interest of space, we will not provide a formal statement of the LDP. Intuitively, when $n$ is large enough, the empirical degree distribution behaves as $\mathbf{P}_{n}\left(\boldsymbol{\mu}^{(n)} \approx \boldsymbol{\mu}\right) \asymp$ $e^{-n I_{E R}(\boldsymbol{\mu} ; \beta)}$.

\section{B. A Formal Anomaly Detection Test}

In this section, we consider the problem of evaluating whether a graph $\mathcal{G}$ is normal, i.e., comes from the ER model with a certain set of parameters $\left(\mathcal{H}_{0}\right)$. Let $\boldsymbol{\mu}_{\mathcal{G}}$ be the empirical degree distribution of the graph $\mathcal{G}$ and let $I_{E R}\left(\boldsymbol{\mu}_{\mathcal{G}} ; \beta\right)$ (cf. Def. 2) be the corresponding rate function. We present the following statement of the generalized Hoeffding test for this anomaly detection problem.

\section{Definition 3}

The Hoeffding test [15] is to reject $\mathcal{H}_{0}$ when $\mathcal{G}$ is in the set:

$$
S_{F}^{*}=\left\{\mathcal{G} \mid I_{E R}\left(\boldsymbol{\mu}_{\mathcal{G}} ; \beta\right) \geq \lambda\right\},
$$


where $\lambda$ is a detection threshold.

It can be shown that the Hoeffding test (1) satisfies the Generalized Neyman-Pearson (GNP) criterion [14].

\section{BOtNet Discovery}

The network anomaly detection technique in the previous section can only report an alarm when a botnet exists. In order to learn more about the botnet, we develop the botnet discovery technique described in this section. The first challenge for botnet discovery is that a single abnormal SIG is usually insufficient to infer complete information about a botnet, including the botmasters and the bots in the botnet. As a result, we monitor windows continuously and store all abnormal SIGs in a pool $\mathcal{A}$. The botnet discovery stage is triggered only when $|\mathcal{A}|>p$.

\section{A. Identification of Pivotal Nodes}

We assume a sequence of abnormal SIGs $\mathcal{A}=$ $\left\{\mathcal{G}_{1}, \ldots, \mathcal{G}_{|\mathcal{A}|}\right\}$. Detecting bots directly is non-trivial. Instead, detecting the leaders (botmasters) or targets is much simpler because they are more interactive than normal nodes. Botmasters need to "command and control" their bots in order to maintain the botnet, and bots actively interact with victims in typical DDoS attacks. Both leaders and targets, henceforth referred to as pivotal nodes, are highly interactive. Let $G_{k}^{i j}$ be an indicator of edge existence between node $i$ and $j$ in $\mathcal{G}_{k}$. Then, for $i=1, \ldots, n$,

$$
e_{i}=\frac{1}{|\mathcal{A}|} \sum_{k=1}^{|\mathcal{A}|} \sum_{j=1}^{n} G_{k}^{i j}
$$

represents the amount of interaction of node $i$ with all other nodes in $\mathcal{A}$. Henceforth, $e_{i}$ is referred to as the total interaction measure of node $i$. We present the following definition of pivotal nodes.

\section{Definition 4}

(Pivotal nodes). We define the set of pivotal nodes $\mathcal{N}=$ $\left\{i: e_{i}>\tau\right\}$, where $\tau$ is a threshold.

After identifying pivotal nodes, the problem is equivalent to detecting the community associated with pivotal nodes.

\section{B. Botnet Discovery}

1) Construction of the Social Correlation Graph: Compared to similar approaches in community detection, e.g., the leader-follower algorithm [16], our method takes advantage of not only temporal features (SIG) but also correlation relationships. These relationships are characterized using a graph, whose definition is presented next.

For $i=1, \ldots, n$, let variable $X_{i}$ represent the number of pivotal nodes in $\mathcal{N}$ that node $i$ has interacted with. Let $\rho\left(X_{i}, X_{j}\right)$ be the sample Pearson correlation coefficient between two random variables $X_{i}$ and $X_{j}$. In addition, if the sample standard deviation of either $X_{i}$ or $X_{j}$ equals zero, we let $\rho\left(X_{i}, X_{j}\right)=0$ to avoid division by zero.

\section{Definition 5}

(Social Correlation Graph). The Social Correlation Graph $(S C G) \mathcal{C}=\left(\mathcal{V}, \mathcal{E}_{c}\right)$ is an undirected graph with vertex set $\mathcal{V}$ and edge set $\mathcal{E}_{c}=\left\{(i, j):\left|\rho\left(X_{i}, X_{j}\right)\right|>\tau_{\rho}\right\}$, where $\tau_{\rho}$ is a threshold.

Because the behaviors of the bots are correlated, they are more likely to be connected to each other in the SCG. Our problem is to find an appropriate division of the SCG to separate bots and normal nodes. Our criterion for "appropriate" is related to the well-known concept of modularity in community detection [17], [18], [19].

2) Modularity-based Community Detection: The problem of community detection in a graph amounts to dividing the vertices of a given graph into non-overlapping groups such that connections within groups are relatively dense while those between groups are sparse [18].

The modularity for a given subgraph is defined to be the fraction of edges within the subgraph minus the expected fraction of such edges in a randomized null model. Although it was proposed as the stopping criterion of a method, this measure later inspired a broad range of community detection methods named modularity-maximization methods.

We consider the simple case when there is only one botnet in the network. As a result, we want to divide the nodes into two groups, one for bots and one for normal nodes. Suppose that $s_{i}$ is variable such that $s_{i}=1$ if node $i$ is a bot and $s_{i}=-1$ otherwise. Let $d_{i}^{c}$ be the degree of node $i$ in $\operatorname{SCG} \mathcal{C}=\left(\mathcal{V}, \mathcal{E}_{c}\right)$ for $i=1, \ldots, n$ and let $m^{c}=\frac{1}{2} \sum_{i} d_{i}^{c}$ be the edge number of $\mathcal{C}$. For a partition specified by $\mathbf{s}=$ $\left(s_{1}, \ldots, s_{n}\right)$, its modularity is defined as in [18]

$$
Q(\mathbf{s})=\frac{1}{2 m^{c}} \sum_{i, j=1}^{n}\left(A_{i j}-N_{i j}\right) \delta\left(s_{i}, s_{j}\right),
$$

where $\delta\left(s_{i}, s_{j}\right)=\frac{1}{2}\left(s_{i} s_{j}+1\right)$ is an indicator of whether node $i$ and node $j$ are of the same type. $A_{i j}=$ $1\left(\left|\rho\left(X_{i}, X_{j}\right)\right|>\tau_{\rho}\right)$ is an indicator of the adjacency of node $i$ with node $j . N_{i j}$ is the expected number of edges between node $i$ and node $j$ in a null model. The selection of the null model is empirical, but the most common choice by far is the configuration model [20] in which $N_{i j}=\frac{d_{i}^{c} d_{j}^{c}}{2 m^{c}}$. The optimal division of vertices should maximize the modularity measure (3).

3) Refined Modularity: We introduce two refinements to the modularity measure to make it suitable for botnet detection. First, intuitively, bots should have strong interactions with pivotal nodes and normal nodes should have weak interactions. We want to maximize the difference. As a result, our objective considers nodes' interaction to the pivotal nodes. Let

$$
r_{i}=\frac{1}{|\mathcal{A}|} \sum_{k=1}^{|\mathcal{A}|} \sum_{j \in \mathcal{N}} e_{i} G_{k}^{i j}
$$

denote the amount of interaction between node $i$ and pivotal nodes. We refer to $r_{i}$ as pivotal interaction measure of node $i$. Then, $\sum_{i} r_{i} s_{i}$ quantifies the difference between the pivotal 
interaction measure of bots and that of normal nodes. A natural extension for the modularity measure is to include an additional term to maximize $\sum_{i} r_{i} s_{i}$.

Second, the modularity measure is criticized to suffer from low resolution, namely it favors large communities and ignores small ones [21], [22]. The botnet, however, could possibly be small. To address this issue, we introduce a regularization term for the size of botnets. It is easy to obtain that $\sum_{i} \mathbf{1}\left(s_{i}=1\right)=\sum_{i} \frac{s_{i}+1}{2}$ is the number of detected bots. Thus, our refined modularity measure is

$$
\begin{aligned}
Q_{d}(\mathbf{s})= & \frac{1}{2 m^{c}} \sum_{i, j \in \mathcal{V}}\left(A_{i j}-\frac{d_{i}^{c} d_{j}^{c}}{2 m^{c}}\right) s_{i} s_{j} \\
& +w_{1} \sum_{i} r_{i} s_{i}-w_{2} \sum_{i} \frac{s_{i}+1}{2}
\end{aligned}
$$

where $w_{1}$ and $w_{2}$ are appropriate weights.

The two modifications also influence the results of isolated nodes with degree 0 , which possibly exist in SCGs. By Def. 5. a node is isolated if its sample deviation is zero or its correlations with other nodes are small enough. The placement of isolated nodes, however, does not influence the traditional modularity measure, resulting in arbitrary community detection results [18]. This limitation is addressed by the two additional terms. If node $i$ is isolated and $r_{i}=0$, then $s_{i}=-1$ in the solution because of the regularization term $w_{2} \sum_{i} \frac{s_{i}+1}{2}$. On the contrary, if $r_{i}$ is large enough, $s_{i}=1$ in the solution because of the term $w_{1} \sum_{i} r_{i} s_{i}$.

\section{Relaxation of the Optimization Problem}

The modularity-maximization problem has been shown as being NP-complete [23], [24]. The existing algorithms for this problem can be broadly categorized into two types: $(i)$ heuristic methods that solve this problem directly [25], and (ii) mathematical programming methods that relax it into an easier problem first [23], [26]. We follow the second route because it is more rigorous.

We define the modularity matrix $\mathbf{M}=\left\{M_{i j}\right\}_{i, j=1}^{n}$, where $M_{i j}=\frac{A_{i j}}{2 m^{c}}-\frac{d_{i}^{c} d_{j}^{c}}{\left(2 m^{c}\right)^{2}}$. Let $\mathbf{s}=\left(s_{1}, \ldots, s_{n}\right)$ and $\mathbf{r}=\left(r_{1}, \ldots, r_{n}\right)$, then the modularity-maximization problem becomes

$$
\begin{array}{ll}
\max & \mathbf{s}^{\prime} \mathbf{M} \mathbf{s}+\left(w_{1} \mathbf{r}^{\prime}-\frac{w_{2}}{2} \mathbf{1}^{\prime}\right) \mathbf{s} \\
\text { s.t. } & s_{i}^{2}=1, \quad \forall i .
\end{array}
$$

To make the objective function concave, we introduce a negative multiple of s' Is [26], leading to:

$$
\begin{array}{ll}
\max & \mathbf{s}^{\prime}(\mathbf{M}-\sigma \mathbf{I}) \mathbf{s}+\left(w_{1} \mathbf{r}^{\prime}-\frac{w_{2}}{2} \mathbf{1}^{\prime}\right) \mathbf{s} \\
\text { s.t. } & s_{i}^{2}=1, \quad \forall i,
\end{array}
$$

where $\sigma$ is a positive scalar. Notice that the objective of (7) is equivalent to that of (6) because $\mathbf{s}^{\prime} \mathbf{I} \mathbf{s}=n s_{i}^{2}=n$ is ensured by the constraint. We can choose $\sigma$ large enough so that $\mathbf{M}-\sigma \mathbf{I}$ is negative definite. This modification induces no extra computational cost. Although the feasible domain of the revised problem is still non-convex, the objective is concave now. (7) is a typical non-convex Quadratically Constrained Quadratic Programming (QCQP) [27]. Let $\mathbf{S}=\mathbf{s s}^{\prime}, \mathbf{P}_{0}=$ $\mathbf{M}-\sigma \mathbf{I}$, and $\mathbf{q}_{0}=w_{1} \mathbf{r}-\frac{w_{2}}{2} \mathbf{1}$. We can relax problem (7) to

$$
\begin{array}{ll}
\max & \operatorname{Tr}\left(\mathbf{S P}_{0}\right)+\mathbf{q}_{0}^{\prime} \mathbf{s} \\
\text { s.t. } & {\left[\begin{array}{lr}
\mathbf{S} & \mathbf{s} \\
\mathbf{s}^{\prime} & 1
\end{array}\right] \succeq 0,} \\
& S_{i i}=1, \quad \forall i .
\end{array}
$$

The problem above is a Semidefinite Programming problem $(S D P)$ and produces an upper bound on the optimal value of the original problem [27]. It is well known that SDP is polynomially solvable and many solvers (CSDP [28], SDPA [29]) are available.

1) Randomization: The SDP relaxation (8) provides an optimal solution together with an upper bound on the optimal value of problem (7). However, the solution of the SDP relaxation (8) may not be feasible for the original problem (7). To generate feasible solutions we use a randomization technique.

If $\left(\mathbf{S}^{*}, \mathbf{s}^{*}\right)$ is the optimal solution of the relaxed problem, then $\mathbf{S}^{*}-\mathbf{s}^{*} \mathbf{s}^{*^{\prime}}$ can be interpreted as a covariance matrix. If we pick $\mathbf{x}=\left(x_{1}, \ldots, x_{n}\right)$ as a Gaussian random vector with $\mathbf{x} \sim \mathcal{N}\left(\mathbf{s}^{*}, \mathbf{S}^{*}-\mathbf{s}^{*} \mathbf{s}^{*^{\prime}}\right)$, then $\mathbf{x}$ "solves" the non-convex QCQP in (7) "on average" over this distribution. As a result, we can draw samples $\mathbf{x}$ from this normal distribution and simply obtain feasible solutions by taking $\hat{\mathbf{x}}=\operatorname{sgn}(\mathbf{x})$. We sample 10,000 points and pick the point that maximizes $f(\mathbf{x})=\mathbf{x}^{\prime}(\mathbf{M}-\sigma \mathbf{I}) \mathbf{x}+\left(w_{1} \mathbf{r}-\frac{w_{2}}{2} \mathbf{1}\right)^{\prime} \mathbf{x}$.

\section{EXPERIMENTAL RESUlTS}

In this section, we apply our network anomaly detection approach to real-world traffic. Meanwhile, we also compare the performance of our botnet discovery approach, a modularity-based community detection technique, with existing community detection techniques.

\section{A. Description of Dataset}

In this paper, we mix some real-world botnet traffic with some real-world background traffic. For the real-world botnet traffic, we use the "DDoS Attack 2007" dataset by the Cooperative Association for Internet Data Analysis (CAIDA) [30]. It includes traces from a Distributed Denialof-Service (DDoS) attack on August 4, 2007. The DDoS attack attempts to block access to the targeted server by consuming computing resources on the server and by consuming all of the bandwidth of the network connecting the server to the Internet.

The total size of the dataset is $21 \mathrm{~GB}$ and the dataset covers about one hour (20:50:08 UTC to 21:56:16 UTC). These dataset only contains attacking traffic to the victim; all other traffic, including the $\mathrm{C} \& \mathrm{C}$ traffic, has been removed by the creator of the dataset. The dataset consists of two parts. The first part is the traffic when the botnet initiates the attack (between 20:50 UTC and 21:13 UTC). In the initiating stage, the bots probe whether they can reach the victim in order to determine the set of nodes that should participate in the attack. The traffic of the botnet during this period is small, 
thus, it is very challenging to detect it using only network load. The second part is the attack traffic which starts around 21:13 UTC when the network load increases rapidly (within a few minutes) from about $200 \mathrm{~Kb} / \mathrm{s}$ to about $80 \mathrm{Mb} / \mathrm{s}$. With this significant change of transmission rate, it is trivial to detect botnets when the attack starts (after 21:13 UTC). In this paper, we select a 5-minutes segment from the first part, i.e., during the time when the botnet initiates the attack. The total number of bot IP addresses in the selected traffic is 136 .

For the background traffic, we use trace 6 in the University of Twente traffic traces data repository (simpleweb) [31]. This trace was measured in a $100 \mathrm{Mb} / \mathrm{s}$ Ethernet link connecting an educational organization to the Internet. This is a relatively small organization with around 35 employees and a little over 100 students working and studying at this site (the headquarters of this organization). There are 100 workstations at this location which all have $100 \mathrm{Mbit} / \mathrm{s}$ LAN connection. The core network consists of a $1 \mathrm{Gbit} / \mathrm{s}$ connection. The recordings took place between the external optical fiber modem and the first firewall. The measured link was only mildly loaded during this period. The background traffic we choose lasts for 3,600 seconds. The botnet traffic is mixed with background traffic between 2,000 and 2,300 seconds.

\section{B. Results of Network Anomaly Detection}

We divide the mixed traffic into 10-second windows and create a sequence of 360 SIGs. Fig. 2. A shows the detection results. The blue "+" markers indicate the value of $I_{E R}\left(\boldsymbol{\mu}_{i} ; \hat{\beta}\right)$ for each window $i, i=1, \ldots, 360$, where $\boldsymbol{\mu}_{i}$ is the empirical degree distribution of SIG $i$ and $\hat{\beta}$ is estimated from the SIGs created using only background traffic. The red dash line shows the threshold $\lambda=0.18$, which can be set to constrain the false alarm rate below a desirable value. According to rule (1), there are 36 abnormal SIGs, namely $|\mathcal{A}|=36$. There are 30 SIGs that have botnet traffic and 29 SIGs are correctly identified. SIG no. 20 corresponding to the time range $[2000 s, 2010 s]$ is missed. Being the start of the botnet traffic, this range has very low botnet activity, which may explain the miss-detection. In addition, there are two groups of false alarms -3 false alarms around 3,000s and 4 false alarms around 3,500s. Fig. 2. B shows the Receiver Operating Characteristic (ROC) curve of the detection rule (1).

\section{Results of Botnet Discovery}

The botnet discovery stage aims to identify bots based on the information in $\mathcal{A}$. The first step is to identify a set of pivotal nodes. Recall that the total interaction measure $e_{i}$ in (2) quantifies the amount of interaction in $\mathcal{A}$ of node $i$ with other nodes. The set of pivotal nodes is $\mathcal{N}=$ $\left\{i: e_{i}>\tau\right\}$, where $\tau$ is a prescribed threshold. Let $e_{\max }$ be the maximum total interaction measure of all nodes and $\mathcal{S}_{e}^{N o r m}=\left\{e_{i} / e_{\max }: i=1, \ldots, n\right\}$ be the normalized set of total interaction measures. Fig. 3 plots $\mathcal{S}_{e}^{\text {Norm }}$ in descending order and in log-scale for the $y$-axis. Each blue "+" marker represents one node. The blue curve in Fig. 3 , being quite
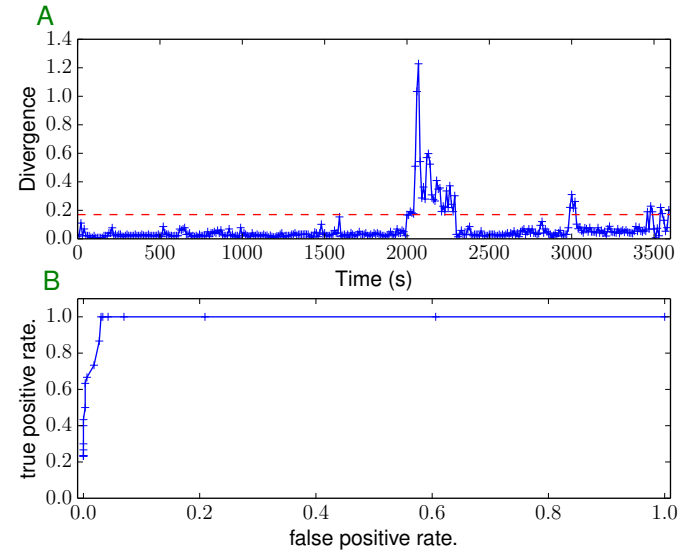

Fig. 2. Figure A shows the rate function value $I_{E R}\left(\boldsymbol{\mu}_{i} ; \hat{\beta}\right)$ for each window $i$. The $x$-axis plots the starting time of each window. The background traffic lasts for 3,600 seconds and the botnet traffic is added between 2,000 and 2,300 seconds. Figure B shows the ROC curve. The $x$-axis plots the false alarm rate and the $y$-axis the true positive rate.

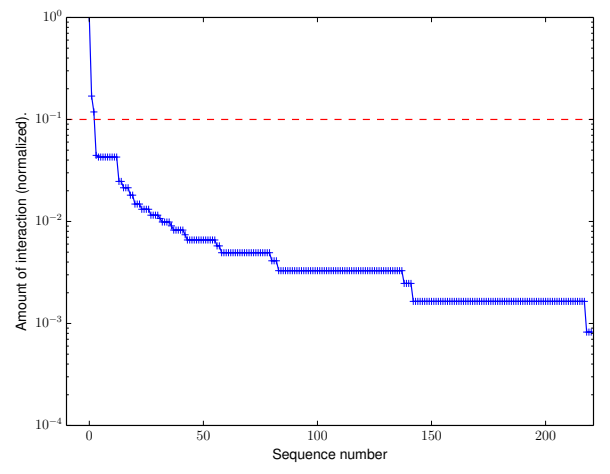

Fig. 3. Sorted amount of interaction in $\mathcal{A}$ defined by 2. $y$-axis is in log-scale.

steep, clearly indicates the existence of influential pivotal nodes. The red dash line in Fig. 3 plots the selected threshold $\tau$, which results in 3 pivotal nodes. Only one pivotal node belongs to the botnet. The other two pivotal nodes are active normal nodes. These two falsely detected pivotal nodes correspond to the two false-alarm groups described in Section V-B

Our dataset has 396 nodes, including 136 bots and 260 normal nodes. Among the 396 nodes, only 213 nodes have positive sample standard deviations. Let $\mathcal{V}_{p}$ be the set of all nodes with positive sample standard deviations, Fig. 4 plots the correlation matrix of these nodes. We can easily observe two groups from Fig. 4 .

We calculate the SCG $\mathcal{C}$ using Def. 5 and threshold $\tau_{\rho}=$ 0.3. In the SCG $\mathcal{C}$, there are 191 isolated nodes with degree zero. The subgraph formed by the remaining 205 nodes has two connected components (Fig. 5.A). Fig. 5.A plots normal nodes as blue circles and bots as red squares. Although the bots and the normal nodes clearly belong to different communities, the two communities are not separated in the narrowest part of the graph. Instead, the separating line is 

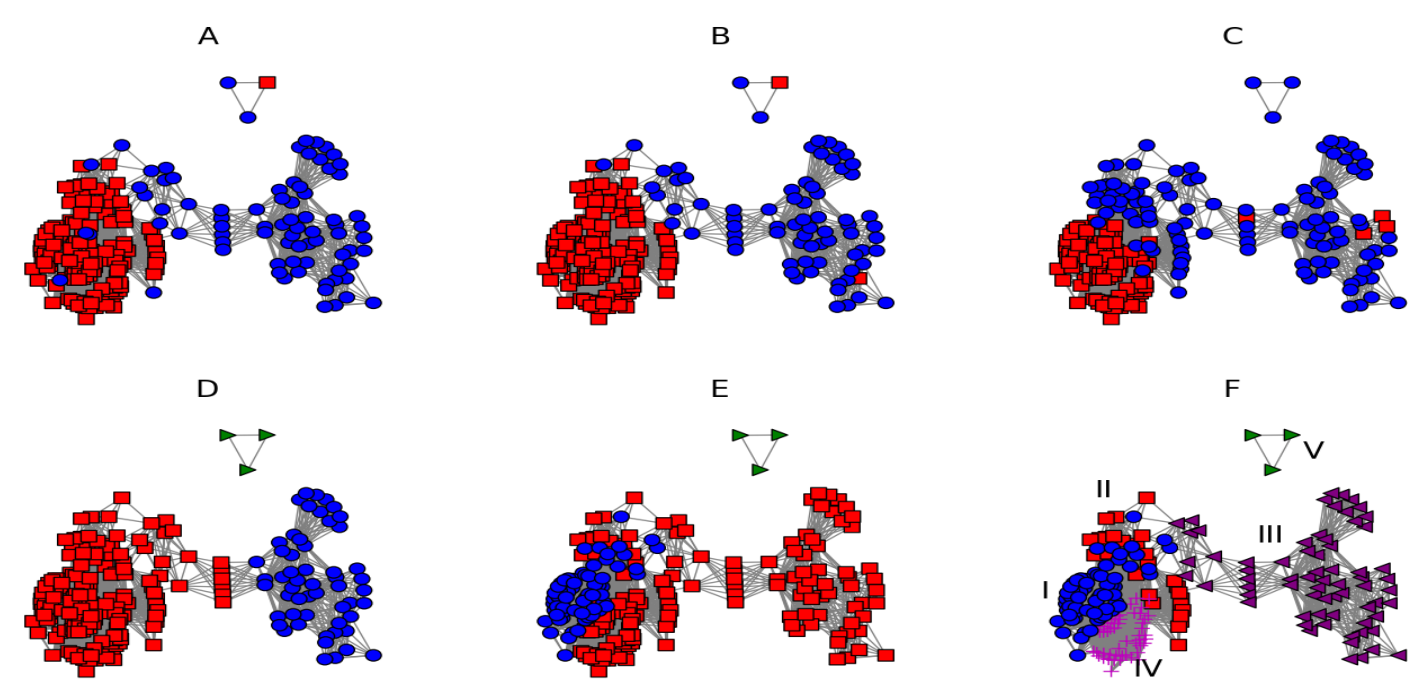

Fig. 5. Comparison of different community detection techniques on SCG. Fig. A shows the ground-truth communities of bots and normal nodes. Fig. $\mathrm{B}$ is the result of our botnet discovery approach. Fig. C is the result of the vector programming method proposed by Agarwal et al. [23]. Fig. D is the result of the walktrap method [32] with three communities. Fig. E is the result of Newman's leading eigenvector method [19] with 3 communities. Fig. F is the result of the leading eigenvector method with 5 communities. In figure A-C, red squares are bots and blue circles are normal nodes. In figure D-F, red squares indicate the group with highest average pivotal interaction measure, while blue circles indicate the group with the lowest one.

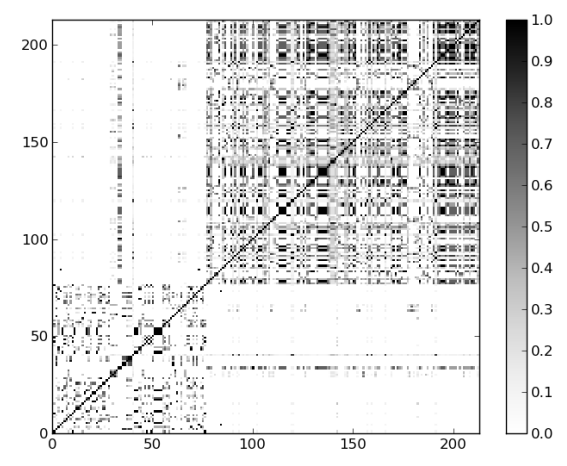

Fig. 4. The correlation matrix $\left[\rho\left(X_{i}, X_{j}\right)\right]_{i \in \mathcal{V}_{p}, j \in \mathcal{V}_{p}}$.

closer to the bots.

We apply our botnet discovery method to $\mathcal{C}$. The result (Fig. 5-B) is very close to the ground truth (Fig. 55-A). As comparison, we also apply other community-detection methods to the 205-node subgraph.

The first method is the vector programming method proposed by Agarwal et al. [23], which is a special case of our method in which $w_{1}=0$ and $w_{2}=0$. This approach, however, misses a number of bots (5-C).

The second method is the walktrap method by Pascal et al. [32], [33], which defines a distance measure for vertices based on a random walk and applies hierarchical clustering [34]. When the desirable number of communities, a required parameter, equals to two, the method outputs the two connected components, a reasonable yet useless result for botnet discovery. To make the results more meaningful, we use walktrap to find three communities and ignore the smallest one that corresponds to the smaller connected components (right triangles in Fig. 5-D). The community with a higher mean of pivotal interaction measure is detected as botnet, and the rest of the nodes are labeled as normal. The walktrap method separates bots and normal nodes in the narrowest part of the graph, a reasonable result from the perspective of community detection (Fig. 5-D). However, a comparison with the ground-truth reveals that a lot of normal nodes are falsely reported as bots.

The third method is the Newman's leading eigenvector method [19], [33], a classical modularity-based community detection method. This method calculates the eigenvector corresponding to the second-largest eigenvalue of the modularity matrix $\mathbf{M}$, namely the leading eigenvector, and lets solution $\mathrm{s}$ be the sign of the leading eigenvector. The method can be generalized for detecting multi-communities [19]. Similar to the walktrap method, the leading eigenvector method reports two connected components as results when the desirable community number is two. We also use this method to find three communities and ignore the smallest one. Again, the community with higher mean of pivotal interaction measure is detected as the botnet.

Different from previous methods, the eigenvector method makes completely wrong prediction of the botnet. The community whose majority are bots (blue circles in Fig. 5-E) is wrongly detected as the normal part and the community formed by the rest of the nodes is wrongly detected as the botnet. Despite being part of the real botnet, the community of blue circles in Fig. 5.E actually has lower mean of pivotal interaction measure, i.e., less overall communication with pivotal nodes.

After dividing the SCG $\mathcal{C}$ into five communities using the leading eigenvector approach for multi-communities [19], we observe that the botnet itself is heterogeneous and divided into three groups. Both the group with the highest mean of pivotal interaction measure (Group II in Fig. 5-F) and the group with the lowest mean (Group I in Fig. 5.F) are part 
of the botnet.

Because of the heterogeneity, some groups of the botnet may be misclassified. On the one hand, the leading eigenvector method wrongly separates Group I from the rest as a single community, and merges Group II \& IV with the normal part (Group III). Because Group I has the lowest pivotal interaction measure, it is wrongly detected as normal, causing Group II, III, IV to be detected as the botnet. On the other hand, the vector programming method wrongly detects a lot of nodes in Group II, which should be bots, as normal nodes.

By taking the pivotal interaction measure into consideration, the misclassification can be avoided. In our formulation of refined modularity (5), the term $w_{1} \sum_{i} r_{i} s_{i}$ maximizes the difference of the pivotal interaction measure of the botnet and that of the normal part. Owing to this term, our method makes little mistake for nodes in Group II since they have high pivotal interaction measures.

\section{Conclusion}

In this paper, we propose a novel method of botnet detection that analyzes the social relationships, modeled as Social Interaction Graphs (SIGs) and Social Correlation Graphs (SCGs), of nodes in the network. Compared to previous methods, our method has following novelties. First, our method applies social network analysis to botnet detection and can detect botnets with sophisticated C\&C channels. Second, our method can be generalized to more types of networks, such as email networks and biological networks [35], [36]. Third, we propose a refined modularity measure that is suitable for botnet detection. The refined modularity also addresses some limitations of modularity.

\section{REFERENCES}

[1] "DDoS Protection Whitepaper," 2012, http://www.neustar.biz/ enterprise/resources/ddos-protection/ddos-attacks-survey-whitepaper\# UtwNR7Uo70o

[2] N. Daswani and M. Stoppelman, "The anatomy of Clickbot.A," in Proceedings of the first conference on First Workshop on Hot Topics in Understanding Botnet, 2007.

[3] Z. Gyongyi and H. Garcia-Molina, "Web spam taxonomy," in First international workshop on adversarial information retrieval on the web (AIRWeb 2005), 2005.

[4] W. T. Strayer, R. Walsh, C. Livadas, and D. Lapsley, "Detecting botnets with tight command and control," in Proceedings of 200631 st IEEE Conference on Local Computer Networks. IEEE, 2006, pp. 195-202.

[5] X. Su and D. Zhang, "Botnet detecting method based on clustering flow attributes of command and control communication channel," Dianzi Yu Xinxi Xuebao(Journal of Electronics and Information Technology), vol. 34, no. 8, pp. 1993-1999, 2012.

[6] J. Binkley and S. Singh, "An algorithm for anomaly-based botnet detection," Proceedings of USENIX Steps to Reducing Unwanted Traffic on the Internet Workshop (SRUTI), pp. 43-48, 2006.

[7] J. Goebel and T. Holz, "Rishi: Identify bot contaminated hosts by IRC nickname evaluation," in Proceedings of the first conference on First Workshop on Hot Topics in Understanding Botnets. Cambridge, MA, 2007, p. 8.

[8] G. Gu, J. Zhang, and W. Lee, "BotSniffer: Detecting botnet command and control channels in network traffic," in Proceedings of 15th Annual Network and Distributed System Security Symposium, 2008.

[9] Z. Bu, P. Bueno, R. Kashyap, and A. Wosotowsky, "The New Era of Botnets," White paper from McAfee, 2010, https://www.botnets.fr/ images/b/b5/Wp-new-era-of-botnets.pdf

[10] R. Lemos, "Bot software looks to improve peer-age," 2006, http:// www.securityfocus.com/news/11390
[11] A. Singh, "Social Networking for Botnet Command and Control," Ph.D. dissertation, San Jose State University, 2012.

[12] Y. Al-Hammadi and A. Abdulla, "Behavioural Correlation for Malicious Bot Detection," Ph.D. dissertation, University of Nottingham, 2010.

[13] S. Mukherjee, "Large deviation for the empirical degree distribution of an Erdos-Renyi graph," arXiv preprint arXiv:1310.4160, pp. 1-23, 2013.

[14] A. Dembo and O. Zeitouni, Large Deviations Techniques and Applications, 2nd ed. Springer, 1998.

[15] W. Hoeffding, "Asymptotically optimal tests for multinomial distributions," Annals of Mathematical Statistics, vol. 36, pp. 369-401, 1965.

[16] D. Shah and T. Zaman, "Community detection in networks: The leader-follower algorithm," arXiv preprint arXiv:1011.0774, pp. 1-13, 2010.

[17] M. Newman, "Fast algorithm for detecting community structure in networks," Physical Review E, vol. 69, no. 6, p. 066133, 2004.

[18] — "Detecting community structure in networks," The European Physical Journal B - Condensed Matter, vol. 38, no. 2, pp. 321-330, 2004.

[19] - "Finding community structure in networks using the eigenvectors of matrices," Physical Review E, vol. 74, no. 3, p. 036104, 2006.

[20] M. Molloy and B. Reed, "A critical point for random graphs with a given degree sequence," Random structures and algorithms, vol. 6, no. 2-3, pp. 161-180, 1995.

[21] S. Fortunato and M. Barthelemy, "Resolution limit in community detection," Proceedings of the National Academy of Sciences of the United States of America, vol. 104, no. 1, pp. 36-41, 2007.

[22] A. Lancichinetti and S. Fortunato, "Limits of modularity maximization in community detection," Physical Review E, vol. 84, no. 6, p. 066122 , 2011.

[23] G. Agarwal and D. Kempe, "Modularity-maximizing graph communities via mathematical programming," The European Physical Journal $B$, vol. 66 , no. 3, pp. 409-418, Nov. 2008.

[24] U. Brandes, D. Delling, M. Gaertler, R. Gorke, M. Hoefer, Z. Nikoloski, and D. Wagner, "On modularity clustering," IEEE Transactions on Knowledge and Data Engineering, vol. 20, no. 2, pp. 172-188, 2008.

[25] J. Duch and A. Arenas, "Community detection in complex networks using extremal optimization," Physical Review E, vol. 72, no. 2, p. 027104, 2005.

[26] E. Y. K. Chan and D.-Y. Yeung, "A convex formulation of modularity maximization for community detection," Proceedings of the TwentySecond international joint conference on Artificial Intelligence, vol. 3 , pp. 2218-2225, 2011.

[27] A. D'Aspremont and S. Boyd, "Relaxations and randomized methods for nonconvex QCQPs," pp. 1-16, 2003, http://web.stanford.edu/class/ ee364b/lectures/OLDrelaxations.pdf

[28] B. Borchers, "CSDP, A C library for semidefinite programming," Optimization Methods and Software, vol. 1, no. 1, pp. 1-10, 1999.

[29] K. Fujisawa, M. Kojima, K. Nakata, and M. Yamashita, "SDPA SemiDefinite Programming Algorithm," Department of Mathematical and Computing Science, Tokyo Institute of Technology, Tech. Rep. B-308, 1995, http://www.is.titech.ac.jp/ kojima/articles/b-308.ps.Z

[30] "The CAIDA UCSD "DDoS Attack 2007" Dataset," CAIDA, 2013, http://www.caida.org/data/passive/ddos-20070804_dataset.xml

[31] R. R. R. Barbosa, R. Sadre, A. Pras, and R. van de Meent, "Simpleweb/university of twente traffic traces data repository," http://eprints.eemcs.utwente.n1/17829/, Technical Report TR-CTIT-1019, April 2010.

[32] P. Pons and M. Latapy, "Computing communities in large networks using random walks," Computer and Information Sciences-ISCIS 2005, 2005.

[33] G. Csardi and T. Nepusz, "The igraph software package for complex network research," InterJournal, Complex Systems, vol. 1695, no. 5, 2006

[34] J. H. Ward Jr, "Hierarchical grouping to optimize an objective function," Journal of the American Statistical Association, vol. 58, no. 301, pp. 236-244, 1963.

[35] M. Newman, S. Forrest, and J. Balthrop, "Email networks and the spread of computer viruses," Physical Review E, vol. 66, no. 3, p. 35101, 2002

[36] M. Newman, Networks: an introduction. Oxford University Press, 2009. 